\title{
Neurally Adjusted Ventilatory Assist (NAVA): Promises and Challenges
}

\section{Farid Zand ${ }^{1 *}$ and Golnar Sabetian ${ }^{2}$}

${ }^{1}$ Associate Professor of Anesthesia and Critical Care Medicine, Shiraz Anesthesiology and Critical Care Research Center, Shiraz University of Medical Sciences, Shiraz, Iran

${ }^{2}$ Assistant Professor of Anesthesia, Fellowship of Critical Care Medicine, Shiraz Anesthesiology and Critical Care Research Center, Shiraz University of Medical Sciences, Shiraz, Iran

Since the introduction of mechanical ventilation, modes of mechanical ventilation have evolved from volume or pressure-targeted, time cycled modes to patient cycled modes [1-3]. There has been a trend over the years toward the use of ventilatory modes in which some degree of spontaneous respiratory activity is preserved, known as partial ventilator assist. Conventional ventilator modes use drop changes in pressure or flow to trigger or terminate a supporting breath [3].

Patient triggered modes have a number of distinct advantages over control modes. These modes have been shown to reduce the adverse effects of prolong sedation [4-6], ventilation-perfusion mismatch [1], hemodynamic instability, neuropathy associated with use of neuromuscular blocking agent [7] and ventilator induced diaphragmatic dysfunction [8], while also improving gas exchange [9], but even in the most common partial ventilatory assist mode (pressure support) asynchrony could be seen [10].

Asynchrony increases respiratory muscle load [7] and is associated with longer duration of mechanical ventilation [11], so this is a very important problem.

Neurally adjusted ventilatory assist (NAVA) is a ventilatory mode which provides pressure in proportion to and in synchrony with the patient's Electrical Activity of the diaphragm (EAdi). During NAVA the ventilator is triggered and cycled off and adjusted by the patient's EAdi signal [10-12].

\section{What is EAdi and How Dose NAVA Use It?}

Spontaneous breathing starts with an impulse generated by respiratory center. The impulse is transmitted over the phrenic nerve, which excites the diaphragm. Before the mechanical effect is achieved, the signal is modulated and the muscle response is achieved by chemical coupling. The contraction of the diaphragm will push the dome downwards, creating a negative alveolar pressure and then the gas flows into the lungs.

The efficacy of the respiratory muscle will determine the degree of respiratory center output. In a healthy subject, the low amplitude of diaphragm excitation reflects the fact that the neuroventilatory coupling is very efficient and that about $5 \%$ of maximum capacity is used. In disease, the muscle may not perform as expected, leading to an increased output from the respiratory center with the aim of recruiting additional muscle groups in the diaphragm $[13,14]$.

With NAVA, ventilator support is initiated when the neural drive to the diaphragm begins to increase. As the EAdi progressively increases, the assist increases proportionally and, most importantly, the pressure delivered by the ventilator is cycled off when the EAdi is ended by the respiratory centers.

The NAVA level is the factor by which the Edi signal is multiplied to adjust the amount of assist delivered to the patient, which expresses a type of gain factor, i.e. how many $\mathrm{cm}_{2} \mathrm{O}$ of support the patient will receive for every $\mu \mathrm{V}$ of EAdi. This assist is thus proportional to the patient's Edi and as such, it follows a physiological pattern. The NAVA level is typically set to between 1.0 and $4.0 \mathrm{~cm} \mathrm{H}_{2} \mathrm{O} / \mu \mathrm{V}$. The range of setting is 0 to $15 \mathrm{~cm} \mathrm{H}_{2} \mathrm{O} / \mu \mathrm{V}$.
When the NAVA level is changed, the resulting pressure delivered by the ventilator depends on how the respiratory afferents modulated the neural output to the diaphragm. If the response to an increase in NAVA is not a reduction in the EAdi, the delivered pressure may remain unchanged and the patient and the respiratory muscles are in a "comfort zone". Thus, the response in EAdi to an increase in the NAVA level determines the resulting transpulmonary pressure (Ptp) and whether volume changes or not consequently. During maximal inspirations, when the EAdi is at its highest, the pressure delivered could reach extreme levels that may cause harm to the lung. It is therefore important to determine, with increasing NAVA level whether or not the EAdi has been suppressed, thereby limiting the pressure delivered during maximal inspiration.

\section{Weaning}

The first sign to look for is a decline in EAdi signal with unchanged tidal volumes. This represents an improvement in neuromuscular coupling in the sense that diaphragm performance is unchanged at lower level of stimulation and the patient is now ready to be weaned. We can gradually reduce the NAVA level in steps of $0.1-0.2 \mathrm{~cm} \mathrm{H}_{2} \mathrm{O} /$ $\mu \mathrm{V}$. No EAdi elevation in response to NAVA level reductions is a positive indicator. Minimal peak pressure, acceptable tidal volume, oxygen saturation, minute ventilation and patient comfort should be used as indicators for withdrawal of ventilatory support. Another sign is peak pressure minus PEEP, if this number is $10 \mathrm{~cm} \mathrm{H}_{2} \mathrm{O}$ the pt will be considered to have been ready to be weaned [19]. The simple monitoring of the EAdi signal without using NAVA mode alone may also provide the clinician with important information about the status of diaphragm muscle and to guide ventilator management.

\section{Potential Benefits of NAVA}

With NAVA, the patient's own respiratory demands determine the level of assistance. EAdi signal and NAVA level titration could be used to determine the level of respiratory unloading, so NAVA makes it possible to avoid over or under assistance of the patient and provides a lung protective mode of ventilation in spontaneous breathing [1921].During NAVA, patient and ventilator are synchronized, which may reduce sedation requirements and improves quality of sleep [22-23].

The EAdi signal is a vital sign for detecting neural breathing efforts, so NAVA can be used for monitoring of patient's sedation while breathing spontaneously [19]. A decrease in EAdi amplitude

${ }^{*}$ Corresponding author: Farid Zand, Associate Professor of Anesthesia and Critical Care Medicine, Shiraz Anesthesiology and Critical Care Research Center Shiraz University of Medical Sciences, Shiraz, Iran, E-mail: zandf@sums.ac.ir

Received May 02, 2012; Accepted May 04, 2012; Published May 07, 2012

Citation: Zand F, Sabetian G (2012) Neurally Adjusted Ventilatory Assist (NAVA): Promises and Challenges. J Pulmon Resp Med 2:-118 doi:10.4172/2161105X.1000e118

Copyright: ( 2012 Zand F, et al. This is an open-access article distributed under the terms of the Creative Commons Attribution License, which permits unrestricted use, distribution, and reproduction in any medium, provided the original author and source are credited. 
with maintained tidal volumes signifies an important improvement in respiratory muscle performance. As the patient's condition improves, EAdi amplitude decreases, resulting in a reduction in ventilatordelivered pressure, so NAVA can facilitate physiological weaning $[19,24]$.Since the diaphragm is consistently activated in NAVA, this may reduce disuse atrophy [19-20].

Contra indications to using the NAVA mode include: When the placement of a naso or orogastric catheter is contraindicated and extremely low(less than $2 \mu \mathrm{V}$ ) to no EAdi signal (use of muscle relaxant or excessive sedation).

\section{Cautionary Notes on the Use of the NAVA Mode}

Patients with extremely high $\mathrm{CO}^{2}$ may exhibit uncontrollable high drive breathing pattern. Sepsis and recovering sepsis patients may exhibit inappropriate neuroventilatory coupling. Massive blood transfusions may alter neuroventilatory coupling. Delirium should be treated before the use of NAVA mode and altered anatomy may not yield a reliable EAdi signal. Severe ARDS patients may also exhibit high drive breathing pattern.

\section{Conclusion}

From a theoretical point of view, NAVA is a new exciting mode of ventilation that has been recently introduced into the clinical practice. Its unique superiority is to rely on EAdi to trigger the inspiratory phase and to deliver proportional assist in synchrony with the patient's neural drive on a breath-to-breath basis. Although we found no direct evidence from randomized clinical trials that show better patientventilator synchrony with NAVA has resulted in better meaningful clinical outcomes, it remains a very promising tool both for clinicians and researchers in the field of critical care. On the other hand, both less EAdi catheter cost and more availability on multiple ventilators are needed before widespread application of this mode is anticipated.

\section{References}

1. Putensen C, Rasanen J, Lopez FA, Downs JB (1994) Effect of interfacing between spontaneous breathing and mechanical cycles on the ventilationperfusion distribution in canine lung injury. Anesthesiology 81: 921-930.

2. Sydow M, Burchardi H, Ephraim E, Zielmann S, Crozier TA (1994) Long-term effects of two different ventilator modes on oxygenation in acute lung injury. Comparison of airway pressure release ventilation and volume-controlled inverse ratio ventilation. Am J Respir Crit Care Med 149: 1550-1556.

3. Esteban A, Anzueto A, Alia I, Gordo F, Apezteguia C, et al. (2000) How is mechanical ventilation employed in the intensive care unit? An international utilization review. Am J Respir Crit Care Med 161: 1450-1458.

4. Girard TD, Kress JP, Fuchs BD, Thomason JW, Schweickert WD, et al. (2008) Efficacy and safety of a paired sedation and ventilator weaning protocol for mechanically ventilated patients in intensive care (awakening and breathing controlled trial): a randomized controlled trial. Lancet 371: 126-134.

5. Kress JP, Pohlman AS, O'Connor MF, Hall JB (2000) Daily interruption of sedative infusions in critically ill patients undergoing mechanical ventilation. $\mathrm{N}$ Engl J Med 342: 1471-1477.

6. Schweickert WD, Gehlbach BK, Pohlman AS, Hall JB, Kress JP (2004) Daily interruption of sedative infusions and complications of critical illness in mechanically ventilated patients. Crit Care Med 32: 1272-1276.

7. Garnacho-Montero J, Madrazo-Osuna J, Garcia-Garmendia JL, Ortiz-Leyba C Jimenez-Jimenez FJ, et al. (2001) Critical illness polyneuropathy: risk factors and clinical consequences. A cohort study in septic patients. Intensive Care Med 27: 1288-1296.

8. Vassilakopoulos T, Petrof BJ (2004) Ventilator-induced diaphragmatic dysfunction. Am J Respir Crit Care Med 169: 336-341.

9. Putensen C, Zech S, Wrigge H, Zinserling J, Stuber F, et al. (2001) Long-term effects of spontaneous breathing during ventilatory support in patients with acute lung injury. Am J Respir Crit Care Med 164: 43-49.
10. Breatnach C, Conlon NP, Stack M, Healy M, O'Hare BP (2010) A prospective crossover comparison of neurally adjusted ventilatory assist and pressuresupport ventilation in a pediatric and neonatal intensive care unit population. Ped Crit Care Med 11: 7-11.

11. Chao DC, Scheinhorn DJ, Stearn-Hassenpflug M (1997) Patient-ventilator trigger asynchrony in prolonged mechanical ventilation. Chest 112: 1592-1599.

12. Piquilloud L, Vignaux L, Bialais E, Roeseler J, Sottiaux T, et al. (2011) Neurally adjusted ventilatory assist improves patient-ventilator interaction. Intensive Care Med 37: 263-271.

13. Sinderby C, Navalesi P, Beck J, Skrobik Y, Comtois N, et al. (1999) Neural control of mechanical ventilation. Nat Med 5: 1433-1436.

14. Sinderby C, Beck J, Spahija J, Weinberg J, Grassino A (1998) Voluntary activation of the human diaphragm in health and disease. J Appl Physiol 85 2146-2158.

15. Aldrich T, Sinderby C, McKenzie D, et al. (2002) Electrophysiologic techniques for the assessment of respiratory muscle function. ATS/ERS statement on respiratory muscle testing. Am J Respir Crit Care Med 166: 518-624.

16. Sinderby C, Beck JC, Lindstrom L, Grassino AE (1997) Enhancement of signal quality in esophageal recordings of diaphragm EMG. J Appl Physiol 82: 13701377.

17. Beck J, Sinderby C, Lindstrom L, Grassino A (1996) Influence of bipolar electrode positioning on measurements of human crural diaphragm EMG. $J$ Appl Physiol 81: 1434-1449.

18. Beck J, Campoccia F, Allo JC, Brander L, Brunet F, et al. (2007) Improved synchrony and respiratory unloading by neurally adjusted ventilatory assis (NAVA) in lung-injured rabbits. Pediatr Res 61: 289-294.

19. Colombo D, Cammarota G, Bergamaschi V, De Lucia M, Corte FD, et al. (2008) Physiologic response to varying levels of pressure support and neurally adjusted ventilatory assist in patients with acute respiratory failure. Intensive Care Med 34: 2010- 2018.

20. Sinderby C, Beck J, Spahija J, de Marchie M, Lacroix J, et al. (2007) Inspiratory muscle unloading by neurally adjusted ventilatory assist during maxima inspiratory efforts in healthy subjects. Chest 131: 711-717.

21. Brander L, Leong-Po H, Beck J, Brunet F, Hutchison SJ, et al. (2008) Titration and implementation of neurally adjusted ventilatory assist in critically ill patients. Chest 135: 695-703.

22. Ambrogio C. Coebnick J, Quan SF, Ranieri M, Parthasarathy S (2008) Assessment of sleep in ventilator-supported critically ill patients. Sleep 31 1559-1568.

23. Bosma K, Ferreyra G, Ambrogio C, Pasero D, Mirabella L, et al. (2007) Patientventilator interaction and sleep in mechanically ventilated patients; pressure support versus proportional assist ventilation. Crit Care Med 35: 1048-1054.

24. Spahija J, de Marchie M, Albert M, Bellemare P, Delisle S, et al. (2010) Patientventilator interaction during pressure support ventilation and neurally adjusted ventilatory assist. Crit Care Med 38: 518-526. 